\title{
RESEARCH OF ECOLOGICAL SAFETY AND CHEMICAL COMPOSITIONS OF PHOSPHOLIPIDS OBTAINED FROM LOCAL VEGETABLE OILS AND THEIR MISCELLES
}

\author{
Dilafruz Sagdullaeva ${ }^{1, *}$, A.S. Turaev ${ }^{2}$, Dilnoza Salikhanova ${ }^{3}$, Saidakbar Abdurakhimov ${ }^{4}$, Dilnoza Jumaeva ${ }^{5}$, and Dildora \\ Ruzmetova $^{6}$ \\ ${ }^{1}$ Institute of Bioorganic Chemistry of the Academy of Sciences of the Republic of Uzbekistan, Tashkent, 100170, Uzbekistan \\ ${ }^{2}$ Institute of Bioorganic Chemistry of the Academy of Sciences of the Republic of Uzbekistan, DSc., Prof., Tashkent, 100170, Uzbekistan \\ ${ }^{3}$ Institute of General and Inorganic Chemistry of the Academy of Sciences of the Republic of Uzbekistan, DSc., Prof., Tashkent, 100170, \\ Uzbekistan \\ ${ }^{4}$ Tashkent Institute of Chemical Technology, DSc., Prof., Tashkent, Uzbekistan \\ ${ }^{5}$ Institute of General and Inorganic Chemistry of the Academy of Sciences of the Republic of Uzbekistan, DSc., Tashkent, 100170, \\ Uzbekistan \\ ${ }^{6}$ Institute of General and Inorganic Chemistry of the Academy of Sciences of the Republic of Uzbekistan, Tashkent, 100170, Uzbekistan
}

\begin{abstract}
There is considered the environmental safety and chemical composition of phospholipids obtained from local vegetable oils and miscella. It was established that the complex analysis of phospholipids using modern methods of IR, NMR, and mass spectroscopy made it possible to identify the main types of phospholipids used in cotton oils obtained by pressing and extraction methods. It is displayed that cottonseed oil, regardless of the method of its production, contains a certain amount of gossypol and its derivatives, since it is environmentally unsafe and these surfactants should not be used for food purposes, which gives reason to recommend the isolated phospholipids for use in technical purposes.
\end{abstract}

\section{Introduction}

Today in the world, much attention is paid to expanding the raw material base and the production of biologically active additives (BAA) to the protected area of phospholipids. The main sources of phospholipids for food purposes are vegetable oils, the production of which is increasing from year to year. New properties of phospholipids are revealed, which are of wide practical importance. Phospholipids turn out to be nonionic surfactants, good emulsifiers (lecithin), antioxidants, liquid viscosity reducers, etc. Unfortunately, in the traditional technology of processing vegetable oils, the production of phospholipids is not given due attention, which is evident from the statistical information on this industry. In this aspect, the improvement of the technology for the production of phospholipids from vegetable oils and the expansion of their areas of application are of priority importance. In world practice, the development of the oil and fat industry is aimed at obtaining various fractions (lecithin, cephalin, etc.) of phospholipids for the food, pharmaceutical, and other sectors of the economy, their processing to synthesize new derivatives for medical purposes and the modernization of technology and technology for their extraction and refinement. In world practice, the development of the oil and fat industry is aimed at obtaining various fractions (lecithin, cephalin, etc.) of phospholipids for the food, pharmaceutical, and other sectors of the economy, their processing to synthesize new derivatives for medical purposes and the modernization of technology and technology for their extraction and refinement. In this regard, it is necessary to justify several scientific and technical solutions in the following directions, including the selection of local vegetable oils rich in phospholipids and not containing toxic substances; intensification of the process of obtaining phospholipids from local vegetable oils using unconventional external influences; development of technologies for upgrading phospholipids and their application in various products [1 ]. The intensive development of technology in the chemical, food, pharmaceutical and other industries is closely related to the use of surfactants of various natures, which make it possible to increase the efficiency of the processes carried out, expand the scope of their application and reduce material and energy costs. Phospholipids are one of the important accompanying triacylglycerides of plant components, in particular cotton oils. Their compositions and properties are characterized by the presence of substances that are similar and depend on the quality of the oilseed raw materials and changes occurring both during the extraction process and during the subsequent processing of vegetable oils. Important properties of phospholipids that determine their consumption as an independent product are their surface-active properties,

\footnotetext{
* Corresponding author: salihanova79@mail.ru
} 
polarity, polarizability, as well as the ability to associate and micelle in non-polar and low-polarity solvents. [2].

They are capable of changing phase and energy interactions at the interfaces between the polar and nonpolar phases. The presence of such activity for phospholipids is due to their chemical structure, polarity and polarizability, as well as external factors: temperature, nature of the medium (solvent), concentration and feature (type) at the interface. [3-7].

\section{Research methods}

This article uses standard analytical methods such as IR, NMR and mass spectroscopy. Results and discussion. Significant difficulties in studying the nature and behaviour of phospholipids obtained from vegetable oils are associated with the complexity of their hydration, conditioned by their lability, the affinity of the structure of their molecules with triacylglycerides, as well as their low content in the extracted raw materials. Phospholipids, possessing a pronounced reactivity, hygroscopicity, instability and other properties, significantly change their extraction. Therefore, when choosing their method of obtaining from vegetable oils, it is necessary to ensure the preservation of the native properties of the extracted phospholipids.

It is known that IR spectroscopy makes it possible to identify bonds in phospholipid molecules and thus it is possible to determine the types of phosphorus-containing compounds, in particular those having hydrogen bonds. The absorption of infrared radiation by a substance causes a transition between vibrational levels of the ground electronic state $[8,9]$. There are two main types of molecular vibrations: valence and deformation. The frequency of the absorbed radiation is equal to the characteristic frequency of the valence and deformation vibrations of the corresponding bond. Consequently, when a molecule is irradiated with IR light, only quanta, whose frequencies correspond to the frequencies of valence or deformation vibrations of the bonds of this molecule, will be absorbed. We took the IR spectra of phospholipids in the form of a film (after having previously dissolved the sample in vaseline oil) on a plate made of a material that was transparent in the region under study (for example, $\mathrm{KBr}, \mathrm{NaCl}, \mathrm{CsI}, \mathrm{KCl}$ ). It must be remembered that vaseline oil strongly absorbs at a frequency of 3000-2800 cm-1, 1460 and $1380 \mathrm{~cm}-1$. Besides, the IR spectra of phospholipids can be recorded in the form of solutions [8].

\section{Results and discussion}

To do this, prepare a $1-2 \%$ solution of the test sample of phospholipid $(10-20 \mathrm{mg} / \mathrm{ml})$ in carbon tetrachloride and place it in double $\mathrm{NaCl}$ cuvettes $0.2 \mathrm{~mm}$ thick. In fig. 1-5 show the IR spectra of some phospholipids isolated from vegetable oils. It should be known that the ionic form of phospholipids, intramolecular and intermolecular interactions affect the absorption frequency corresponding to phosphate groups. [10]. While studying the structure of phospholipids, NMR and mass spectroscopy long with IR spectroscopy are used, which is necessary for their final identification.

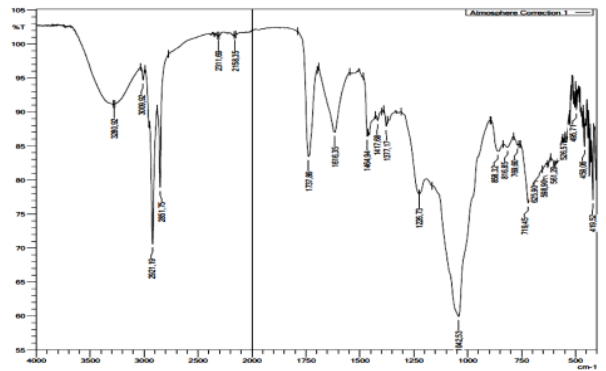

a)

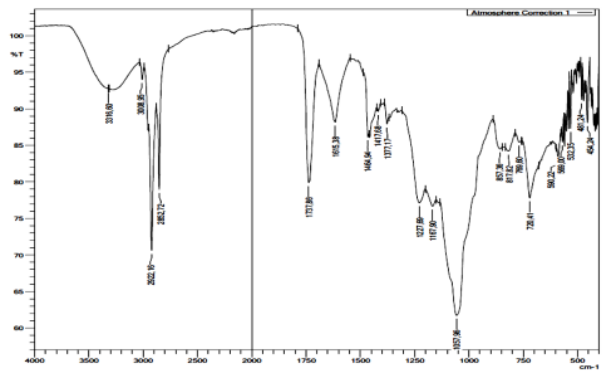

b)

Fig. 1. IR spectra of phospholipids obtained from (a) pressed and (b) extracted cottonseed oil (a -Absorption bands, 1737.86 and 1616.35 , and 1042.53 were found respectively, due to valence vibrations of carbonyl groups, $\mathrm{C}=\mathrm{C}$ and complex ether groups in the IR spectra of the samples; $b$ - In the IR spectra of the samples, absorption bands 1737.86 and 1615.38, as well as 1057.96 , due to valence vibrations of carbonyl groups $\mathrm{C}=\mathrm{C}$ and complex ether groups, respectively, were found).

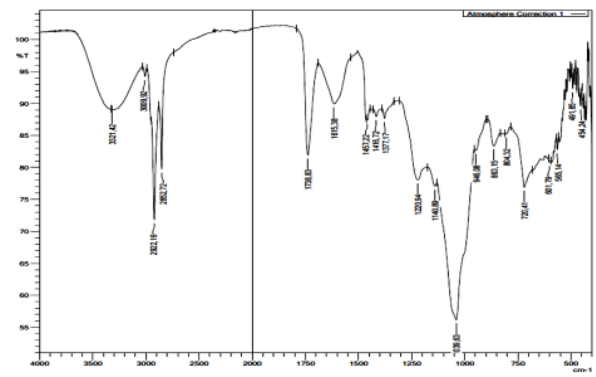

a)

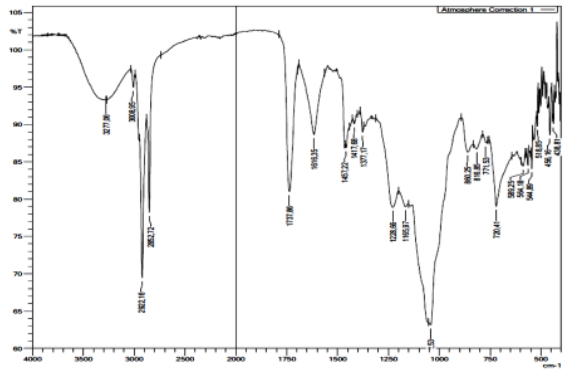

b)

Fig. 2. IR spectra of phospholipids obtained from pressed (a) and extraction (b) cottonseed oil (a - Absorption bands of 1738.83 and 1615.38 were found, as well as 1039.63 due to valence vibrations of carbonyl groups, $\mathrm{C}=\mathrm{C}$ and complex ether groups, respectively, in the IR spectra of the samples; $\mathrm{b}$ The IR spectra show absorption bands at 1737.86 and 1616.35 , and 1057.53 , respectively due to valence vibrations of carbonyl groups, $\mathrm{C}=\mathrm{C}$ and high ether groups). 


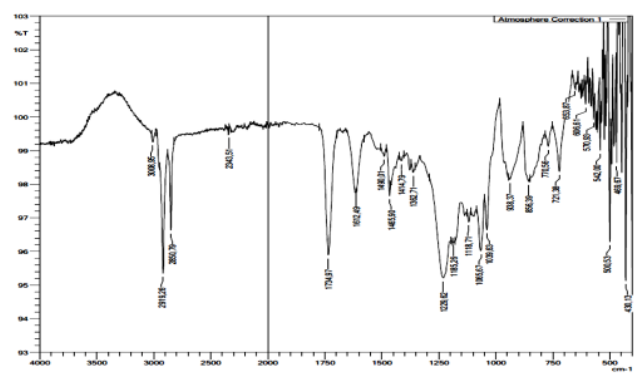

a)

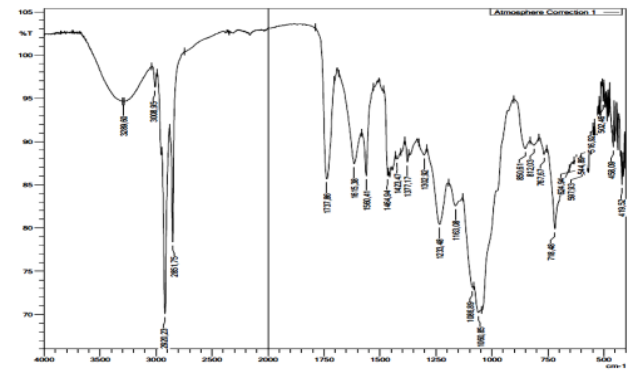

b)

Fig. 3. IR spectra of phospholipids obtained from pressed (a) and extraction (b) cottonseed oilsamples (a - The IR spectra show absorption bands at 1734.97 and 1612.49 and 1229.62 and 1065.67 due to valence vibrations of carbonyl groups, $\mathrm{C}=$ $\mathrm{C}$ and complex ether groups, respective; b- Absorption bands of 1737.86 and 1615.38 , and 1233.48 and 1060.85 , were found, respectively, due to valence vibrations of carbonyl groups, $\mathrm{C}=$ $\mathrm{C}$ and complex ether groups, in the IR spectra of the sample).

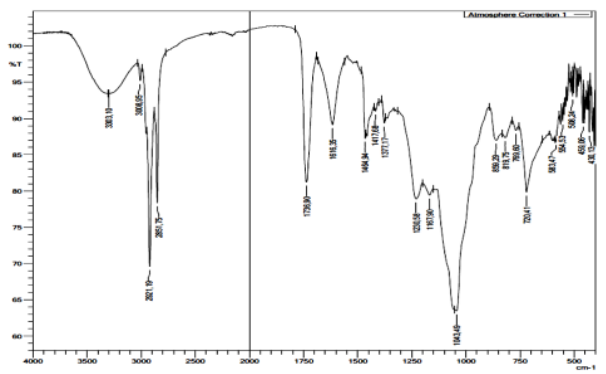

a)

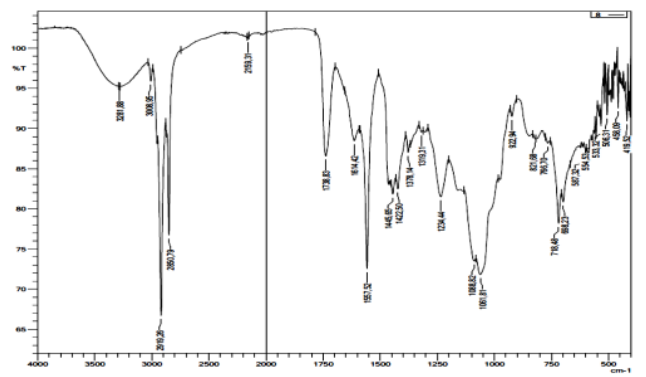

b)

Fig. 4. IR spectra of phospholipids obtained from pressed (a) and extraction (b) cottonseed oil (a - Absorption bands at 1736.90 and $1616.35 ; 1464.94$, and 1043.49 were shown, respectively, due to valence vibrations of carbonyl groups, $\mathrm{C}=$ $\mathrm{C}$ and complex ether groups, in the IR spectra of the samples; $\mathrm{b}$ - Absorption bands of 1738.83 and 1557.52 and 1061.81 were detected, respectively due to valence vibrations of carbonyl groups, $\mathrm{C}=\mathrm{C}$ and complex ether groups in the IR spectra of the samples).

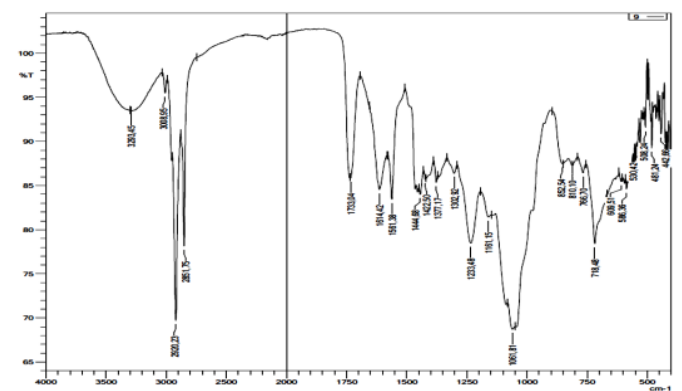

a)

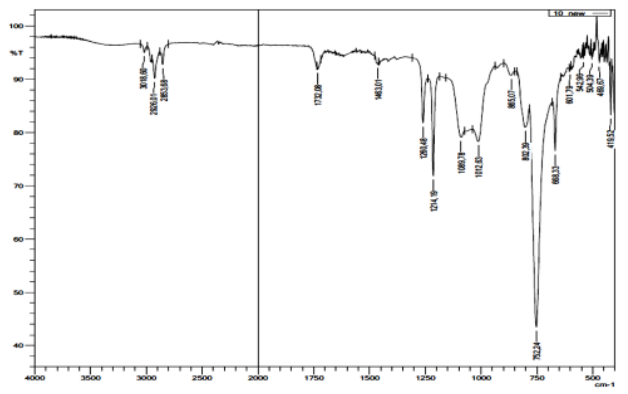

b)

Fig. 5. IR spectra of phospholipids obtained from the press (a) and extraction (b) cottonseed oil (a - Absorption bands of 1733.04 and 1561.38 , and 1238.48 and 1061.81, were found, respectively, due to valence vibrations of carbonyl groups, $\mathrm{C}=$ $\mathrm{C}$ and complex ether groups, in the IR spectra of the sample; $\mathrm{b}$ - Absorption bands of 1732.08 and 1214.19 were found, as well as 1012.63 due to valence vibrations of carbonyl groups, $\mathrm{C}=\mathrm{C}$ and complex ether groups, respectively, in the IR spectra of the samples).

The NMR method, the induction of resonance in atomic nuclei, most often in protons, when irradiated with radio waves in a strong magnetic field $[8,9]$. The position of absorption bands or chemical shifts is measured relative to an arbitrary standard, usually tetramethylsilane (TMS), and is expressed in parts per million of the magnetic field strength ( $\mathrm{ppm})$ :

$$
\delta=\frac{\Delta \vartheta \cdot 10^{6}}{\text { magnetic field frequency, } M \mathrm{~Hz}} ;
$$

where: $\Delta v$-difference between the absorption frequencies of the test sample and the standard, Hz.

If tetramethylsilane has taken as the standard, then the value of its absorption band is conveniently taken as 10 , and the absorption of the test sample is expressed by the $\tau$ (ppm), with $\tau=10-\delta$.

The sample prepared in the same way, for obtaining the IR spectrum. Take NMR spectra of concentrated solutions of individual phospholipid groups (35-50 $\mathrm{mg} / \mathrm{ml}$ ). Aprotic solvents: carbon tetrachloride, deuterochloroform, carbon disulfide or deuterium water has used, $5-10 \%$ tetramethylsilane has added to the test solution as an internal standard in the NMR spectrum. The minimum amount of solution, required to obtain the spectrum is $0.3-1.0 \mathrm{ml}$; in this regard, at least $10-25 \mathrm{mg}$ of phospholipids, required to record the NMR spectrum.

Below are the basic structural formulas of phosphatidylethanolamines (1), phosphatidylcholines (2) and phosphatidylserines 

(e)
(b) (d) (c)
(b) (a)

(g) $\mathrm{CH}_{2} \mathrm{OCOCH}_{2}\left(\mathrm{CH}_{2}\right)_{5} \mathrm{CH}_{2} \mathrm{CH}=\mathrm{CHCH}_{2}\left(\mathrm{CH}_{2}\right)_{6} \mathrm{CH}_{3}$

(f) $\mathrm{CHOCOCH}_{2}\left(\mathrm{CH}_{2}\right)_{5} \mathrm{CH}_{2} \mathrm{CH}=\mathrm{CHCH}_{2}\left(\mathrm{CH}_{2}\right)_{6} \mathrm{CH}_{3}$

(h) $\mathrm{CH}_{2} \mathrm{OPO}_{2}^{-}-\mathrm{OCH}_{2}-\mathrm{CH}_{2}-\mathrm{N}^{+} \mathrm{H}_{3}$

(h) (i) (j)
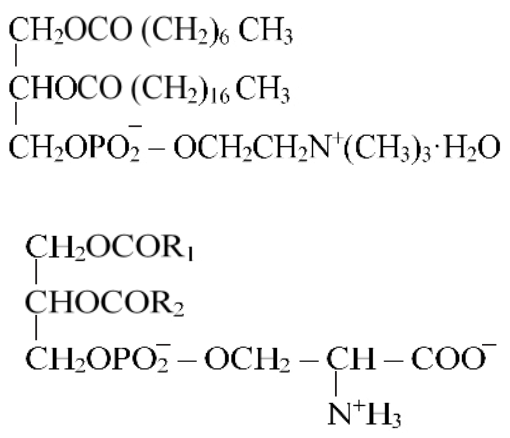

For example, in the structural formula of phosphatidylethanolamine (1), letters denote protons that cause the appearance of peaks in the NMR spectrum. Several characteristic NMR spectra of phospholipids have shown in Fig. 2, a, b, in [8]. For a quantitative analysis of the peak areas, we summarize, a value proportional to the total number of protons in a molecule. The number of protons in any functional group expresses the ratio of the area of a given peak or group of peaks to the total area.

Use of IR and NMR spectroscopy is the most effective method for studying the structure of phospholipids.

Table 1. Chemical shift values of phospholipid compounds.

\begin{tabular}{|c|c|c|c|}
\hline № & Compound or group & con. char. & $\begin{array}{c}\text { Chemical shift } \\
\delta, \text { ppm }\end{array}$ \\
\hline 1 & $\mathrm{CH}_{3}$ & a & $9,00-9,12$ \\
\hline 2 & $\mathrm{CH}_{2}$ & b & $8,70-8,74$ \\
\hline 3 & $-\mathrm{CH}_{2} \mathrm{CH}=$ & d & $7,95-8,08$ \\
\hline 4 & $-\mathrm{CH}_{2} \mathrm{CO}-$ & e & $7,66-7,74$ \\
\hline 5 & $-\mathrm{CH}=\mathrm{CH}-$ & c & $4,60-4,63$ \\
\hline 6 & $\mathrm{CH}_{2} \mathrm{NH}_{2}$ & i & $6,70-6,85$ \\
\hline 7 & $-\mathrm{CH}_{2} \mathrm{~N}^{+}\left(\mathrm{CH}_{3}\right)_{3}$ & - & $6,0-6,18$ \\
\hline 8 & $-\mathrm{N}^{+}\left(\mathrm{CH}_{3}\right)_{3}$ & - & $6,54-6,77$ \\
\hline 9 & $-\mathrm{CH}_{2} \mathrm{OCO}-$ & g & $5,87-5,99$ \\
\hline 10 & $-\mathrm{CH}_{2} \mathrm{OPO}-$ & h & $5,64-5,85$ \\
\hline 11 & $-\mathrm{CHOCO}_{-}$ & f & $4,78-4,80$ \\
\hline 12 & $-{ }^{+} \mathrm{NH}$ & j & $1,50-1,65$ \\
\hline
\end{tabular}

Below is a list of proton resonance signals, which can be used to identify the main groups of phospholipids (Table 1) $[11,8]$.

We have recorded NMR (PMR) spectra of phospholipids obtained from various types of vegetable oils (Fig. 6)

Usually, in the PMR spectra, proton signal broadening is not detected.

According to the literature $[11,8]$ at $1.35 \mathrm{ppm}$ - refer to proton groups. 7.5-field weak, Sp hybrid aromatic hydrocarbons come in. In the PMR spectrum, the signals in samples b) and c) are identical to sample a).

The 2-bond proton signal will last 5.5 lobes.

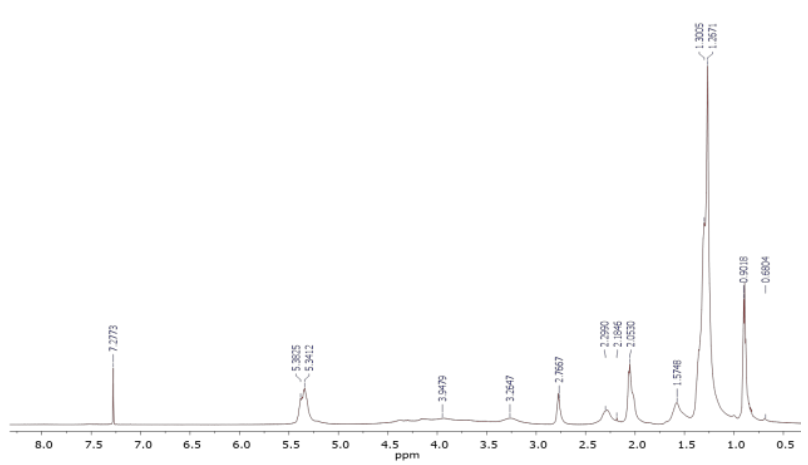

a)

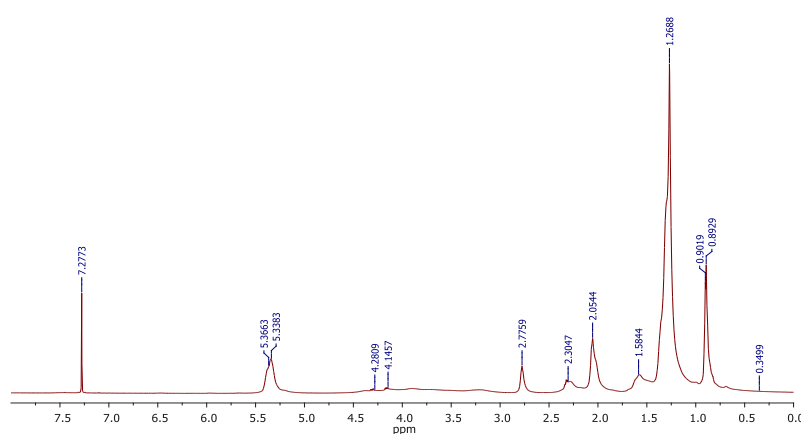

b)

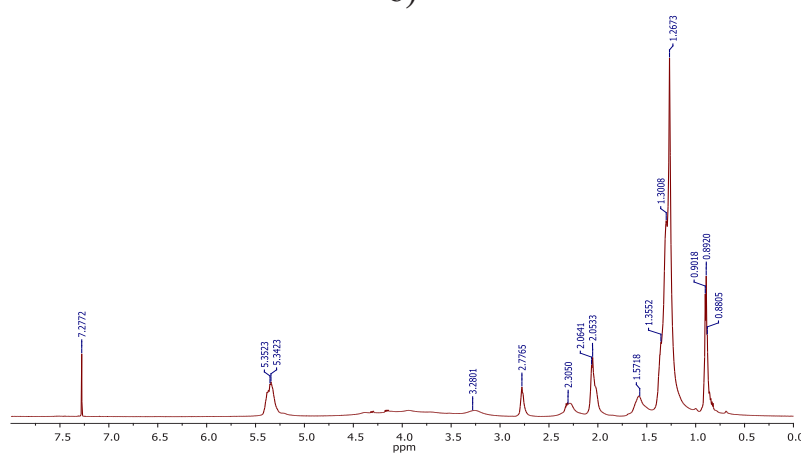

c)

Fig. 6. NMR spectra of phospholipids (a - NMR (PMR) spectroscopy of phospholipids obtained from defatted powder (purified with acetone) hydrated with $4 \%$ distilled water of forepress cottonseed oil (Proton spectrum, spectra C13 and C13-apt additionally obtained; b- NMR (PMR) - spectroscopy of phospholipids obtained by hydration of defatted powder (purified with acetone), forepress oil with $3 \%$ alkali $\mathrm{NaOH}$ (proton spectrum) (additionally obtained spectra C13 and C13apt); c - c) NMR (PMR) - spectroscopy of phospholipids obtained from defatted powder (purified with acetone), hydrated with $0.3 \%$ acetic acid of forepress cottonseed oil (Proton spectrum, additionally obtained spectra $\mathrm{C} 13$ and $\mathrm{C} 13-$ apt)).

Fig. 6 shows that phospholipids obtained from local vegetable oils contain almost all the main groups of surfactants.

The mass spectrum of phospholipids consists of characteristic peaks corresponding to molecular fragments with different ratios of mass to electron charge (w/e), formed as a result of bombardment of a substance with electrons in an ionization chamber and/or as a result of "cracking" during the evaporation of a substance $[11,8]$. The sample preparation technique depends on 
how it is introduced into the mass spectrometer. There are currently 3 ways to introduce the sample:

a) using a flow divider installed at the outlet of the gas-liquid chromatography, which directs part of the flow of the carrier gas with the substance directly to the mass spectrometer [8]. This method is applicable only when working with instruments that combine a mass spectrometer and gas-liquid chromatography. In this case, the sample, as soon as it is eluted from the column of the chromatograph, is automatically introduced into the spectrometer; b) direct introduction into the spectrometer: a few millilitres (3-5) of a concentrated $15-20 \%$ solution of the sample in a volatile inert solvent such as hexane, diethyl ether (free of peroxides) or benzene is introduced with a micropipette into a glass tube with an inner diameter of $2 \mathrm{~mm}$ or into a capillary and evaporated to near dryness in a stream of nitrogen. The sample is placed in an ampoule, which is introduced into the vacuum system of the mass spectrometer. The system is evacuated to remove traces of solvent. The ampoule is introduced through a vacuum lock into the ion source. This technique is used when working with substances with relatively low volatility; sample weight should be 0.1-0.2 mg [12];

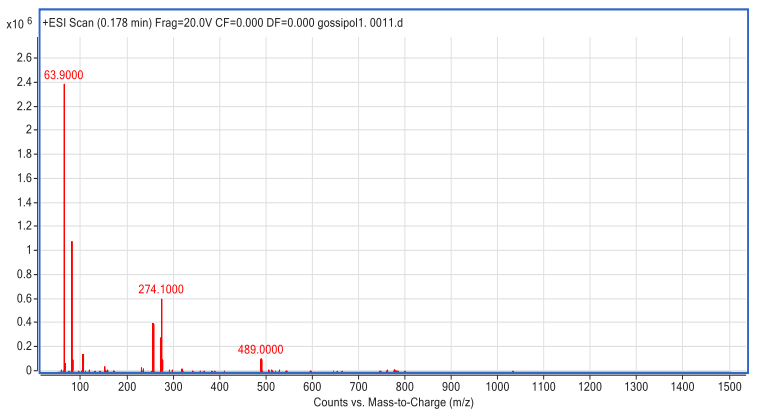

a)

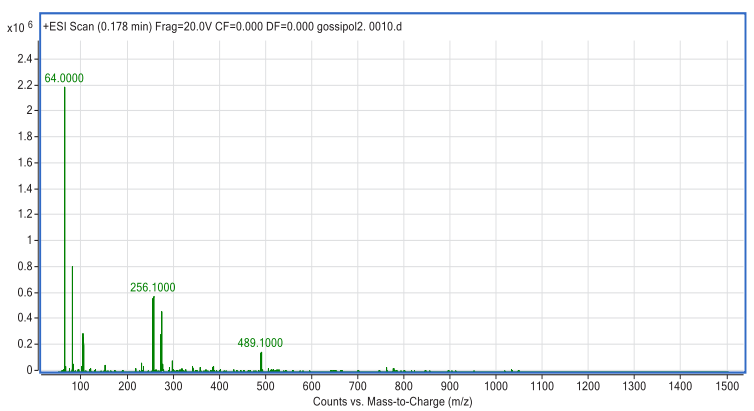

b)

Fig. 7. Mass spectra of phospholipids isolated from the press (a) and extraction (b) cotton oils (a - Obtained fragments of gossypol in phospholipids obtained by the traditional method of hydration of forepress cottonseed oil; $b$ - obtained fragments of gossypol in the conventional sense of hydrated phospholipids of extraction cottonseed oil).

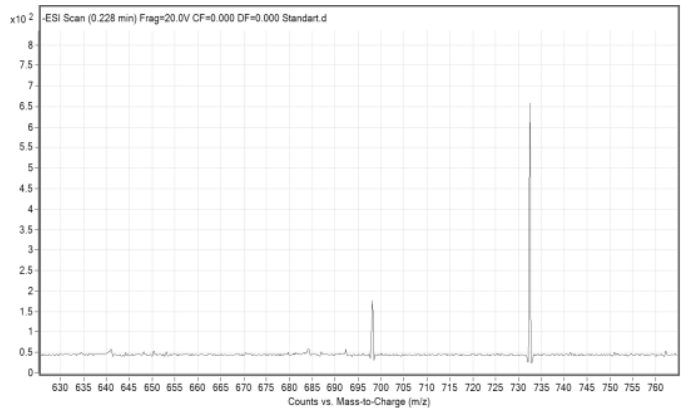

a)

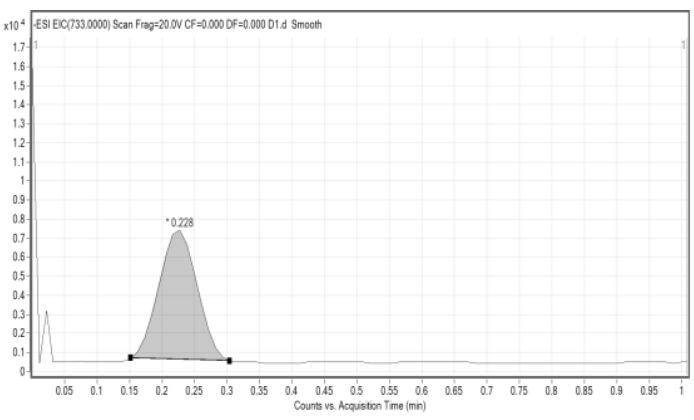

b)

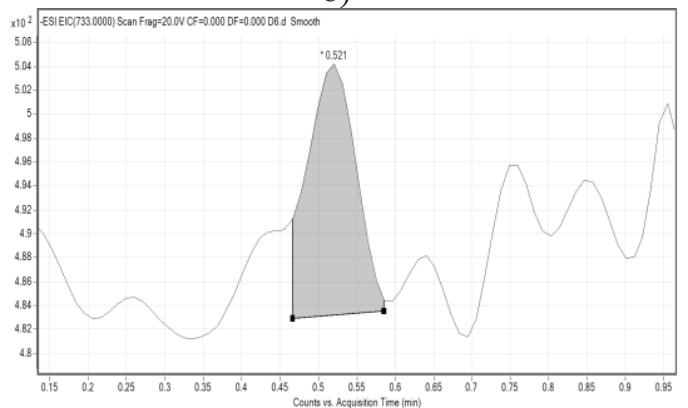

c)

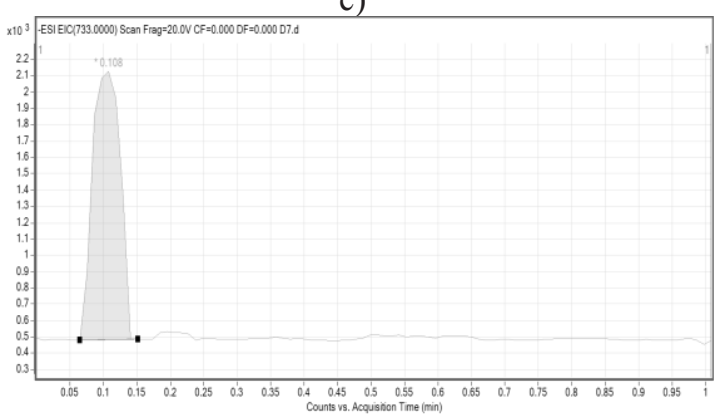

d)

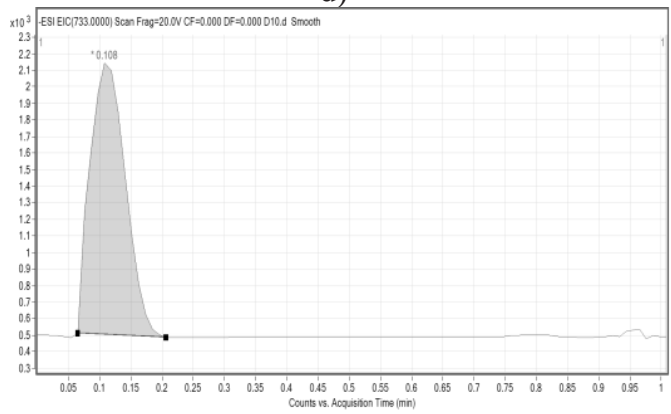

e)

Fig. 8. Mass spectra of phospholipids obtained from various vegetable oils. 
c) a solution of the sample $(0.2-1 \mathrm{mg})$ in one of the above solvents is placed in a $10 \mathrm{ml}$ round-bottom flask with a standard thin section and evaporated in a stream of nitrogen, the flask is connected to the external outlet of the mass spectrometer. Over a period of time, the pressure is reduced and the flask is gradually heated from the beginning (up to $300^{\circ} \mathrm{C}$ and above). As a result, the evaporated sample enters the mass spectrometer through a thin capillary storage device. The mass spectrometric method for the analysis of the decay products of synthetic phospholipids after pyrolysis gas chromatography was described for the first time in the work [12]. Mass spectrometry data for the analysis of synthetic phosphatidylcholines, issues of energy, electrical mechanisms and the energy of adsorption of the adsorbent obtained in the work [13, 14-19]. Highresolution mass spectrometers were used; direct sample injection at a source temperature of $250^{\circ} \mathrm{C}$ and an ionization potential of $70 \mathrm{EV}$. The ions associated with hydrocarbon chains, glycerol and phosphorylcholine esters have been identified. The molecular ion was presumably identified only in the case of dioleoyl glyceryl phosphorylcholine

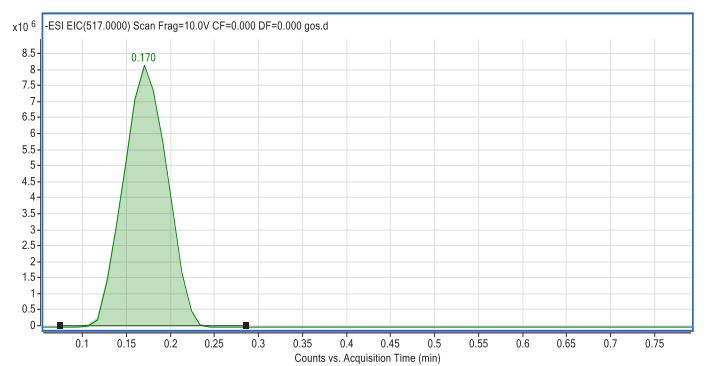

a)

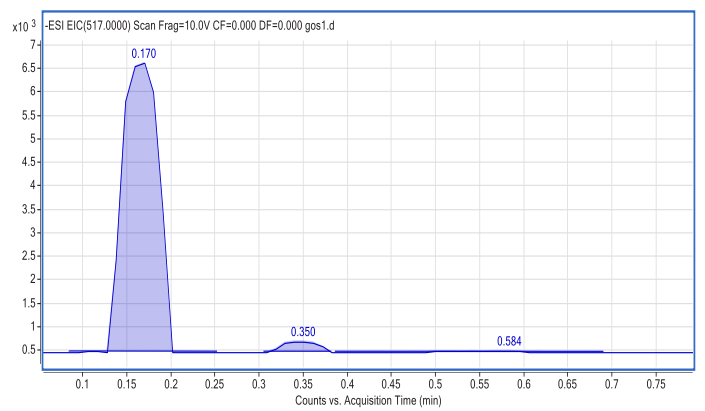

b)

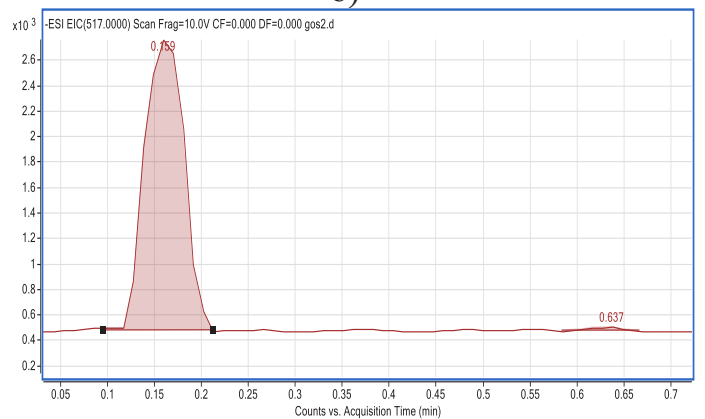

c)

Fig. 9. Mass spectra of gossypol in phospholipids isolated from cotton oils.

\section{Conclusion}

Thus, a comprehensive analysis of phospholipids using modern methods of IR, NMR and mass spectroscopy made it possible to identify the main types of phospholipids contained in cotton oils obtained by pressing and extraction methods. It is shown that cottonseed oil, regardless of the method of its production, contains a certain amount of gossypol and its derivatives, which gives grounds to recommend the isolated phospholipids for use in technical purposes.

\section{References}

1. S. Urinov, D. Sagdullaeva, A. Turaev, S. Abdurakhimov, Advantages of Use of Technical Phospholipides of Cotton Oils for Producinga Lubricant and Anti-corrosion Drilling Mix/International Journal of Advanced Research Science, Engineering and Technology, India, 7 (1), 12444-12448 (January 2020)

2. V. Constantinos, Nikiforidis Biobased, Chemistry and Technology, Wageningen University and Research, Bornse Weillanden 9, P.O. Box 17, 6708WG, Wageningen, the Netherlands DOI: 10.1016/j.cis.2019.102039

3. B. Vijayalakshmi, S.V. Rao, Fatty acid composition of phospholipids in seed oils containing unusual acids, Chemistry and Physics of Lipids, 9 (1), 82-86 (1972)

4. V.I. Martovshuk, T.V. Mgebrishvili, Surface activity of accompanying substances in hexane miscells of sunflower oil, Oil and fat industry, 6 , 14-16 (1978)

5. V.I. Martovshuk, T.V. Mgebrishvili, A.V. Paukova, About interfacial activity and micelle formation of lecithin in non-polar liquids, Proceedings of VNIIZh, 41-45 (1980)

6. K.L. Mittel, P. Mukherjee, L.M. Prince, et all, Micelle formation, stabilization and microemulsions $(597,1980)$

7. P.H. Elworthy, D.S. Mcintosh, The Interaction of water with Lecitin Micelles in Benzene, H. Phys. Chem., 68 (12), 3448-3452 (1964)

8. L.A. Kazatsina, M.B. Kupletskaya, Application of UV, IR, NMR and mass spectroscopy in organic chemistry, Publishing house of Moscow State University $(238,1979)$

9. N.M. Emanuel, Experimental methods of chemical kinetics (M .: Higher School, 171, 1971)

10. M.B. Abramson, W.T. Norton, R. Katzman, Study of ionic structures in phospholipids by infrared spectra, J. Biol. Chem., 240 (6), 23892395 (1986)

11. N.S. Harutyunyan, E.P. Kornena, Phospholipids of vegetable oils (M .: Agropromizdat, 256, 1986)

12. G. Budzikevich, K. Jerassi, D. Ulyams, Interpretation of mass spectra of organic compounds $(323,1966)$

13. R.A. Klein, Mass spectrometry of the phosphatidylcholines: dipalmitoyl, dioleoyl and 
stearoyloleoy glycerylphosphorycholines, J. Lipid Res., 12 (1-2), 123-132 (1971)

14. D. Jumayeva, I. Eshmetov, B. Jumabaev, A. Agzamkhodjayev, Carbon adsorbents on the basis of Brown coal of Angren for cleaning industrial wastewater, Journal of Chemical Technology and Metallurgy, 51 (2), 210-214 (2016)

15. D. Bystrov, O. Toirov, G. Mustafakulova, D. Yakubova, NISS2020: Proceedings of the 3rd International Conference on Networking, Information Systems \& Security, 54, 1-3 (2020) DOI: $10.1145 / 3386723.3387873$

16. D. Bystrov, O. Toirov, S. Giyasov, M. Taniev, U. Sardor, NISS2020: Proceedings of the 3rd International Conference on Networking, Information Systems \& Security, 49, 1-4 (2020) DOI: $10.1145 / 3386723.3387868$

17. O. Toirov, A. Bekishev, S. Urokov, U. Mirkhonov, E3S Web of Conferences, 216, 01116 (2020) DOI: 10.1051/e3sconf/202021601116

18. O. Toirov, K. Alimkhodjaev, N. Pirmatov, A. Kholbutaeva, E3S Web of Conferences, 216, 01119 (2020) DOI: 10.1051/e3sconf/202021601119

19. H. Afrisal, B. Setiyono, M.F. Yusuf, R.M. Suin, O. Toirov, 7th International Conference on Information Technology, Computer, and Electrical Engineering (ICITACEE), 41-46 (2020)

DOI:

10.1109/ICITACEE50144.2020.923922 